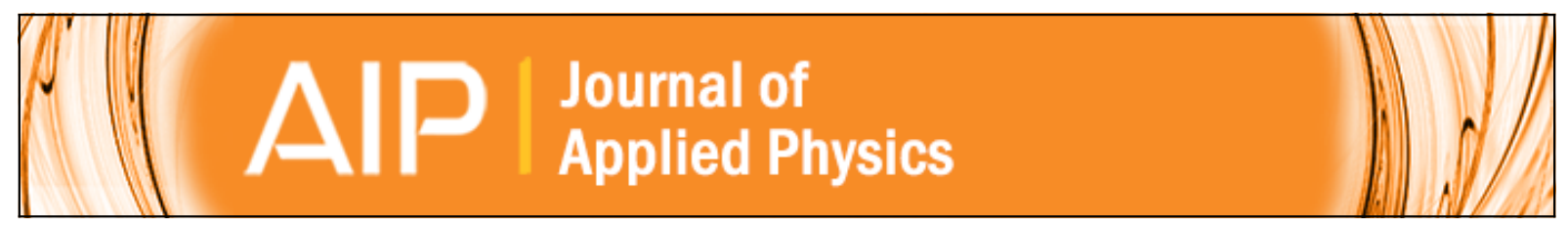

\title{
Limitations on standard procedure of determining internal loss and efficiency in quantum dot lasers
}

Levon V. Asryan

Citation: Journal of Applied Physics 99, 013102 (2006); doi: 10.1063/1.2159072

View online: http://dx.doi.org/10.1063/1.2159072

View Table of Contents: http://scitation.aip.org/content/aip/journal/jap/99/1?ver=pdfcov

Published by the AIP Publishing

\section{Articles you may be interested in}

Effect of internal optical loss on the modulation bandwidth of a quantum dot laser

Appl. Phys. Lett. 100, 131106 (2012); 10.1063/1.3697683

Theoretical study on high-speed modulation of Fabry-Pérot and distributed-feedback quantum-dot lasers: K factor-limited bandwidth and $10 \mathrm{Gbit} / \mathrm{s}$ eye diagrams

J. Appl. Phys. 101, 013108 (2007); 10.1063/1.2407259

Comparison between multilayered InAs quantum dot lasers with different dot densities

Appl. Phys. Lett. 88, 241117 (2006); 10.1063/1.2213520

Optical loss and lasing characteristics of high-quality-factor AIGaAs microdisk resonators with embedded quantum dots

Appl. Phys. Lett. 86, 151106 (2005); 10.1063/1.1901810

Lasing characteristics of InAs quantum-dot lasers on (001) InP substrate

Appl. Phys. Lett. 83, 1704 (2003); 10.1063/1.1606501

MIT LINCOLN

LABORATORY

CAREERS

Discover the satisfaction of innovation and service

to the nation
- Space Control

- Air \& Missile Defense

- Communications Systems \& Cyber Security

- Intelligence, Surveillance and

Reconnaissance Systems
- Advanced
Electronics
- Tactical Systems
- Homeland
Protection
- Air Traffic Control

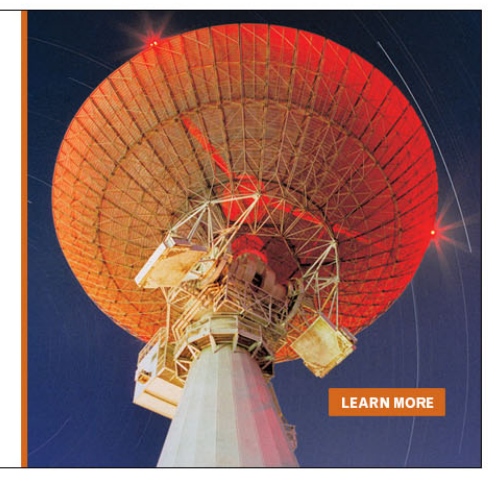




\title{
Limitations on standard procedure of determining internal loss and efficiency in quantum dot lasers
}

\author{
Levon V. Asryan ${ }^{\text {a) }}$ \\ Virginia Polytechnic Institute and State University, Blacksburg, Virginia 24061
}

(Received 24 June 2005; accepted 29 November 2005; published online 12 January 2006)

\begin{abstract}
Limitations are discussed on the use in quantum dot (QD) lasers of the conventional method of determining internal loss coefficient $\alpha_{\text {int }}$ and internal quantum efficiency $\eta_{\text {int }}$ from a measured plot of the reciprocal slope efficiency versus the cavity length $L$. The limitations are imposed by the $L$-dependence of $\alpha_{\text {int }}$ and $\eta_{\text {int }}$ themselves. The effect of internal loss is quantitatively analyzed, which originates from the dependence of $\alpha_{\text {int }}$ on the carrier density, with the latter being $L$-dependent. In short cavities, a plot of the reciprocal slope efficiency versus $L$ can significantly deviate from a straight line, thus limiting the practicality of the standard procedure. For $L$ longer than several hundred $\mu \mathrm{m}$, the limitations are strong in a single-QD-layer laser and moderate in a multiple-QD-layer laser. (C) 2006 American Institute of Physics. [DOI: 10.1063/1.2159072]
\end{abstract}

\section{INTRODUCTION}

Design optimization of diode lasers requires a knowledge and control over a number of parameters and characteristics, ${ }^{1}$ such as the internal absorption loss coefficient $\alpha_{\text {int }}$ (referred to as the internal loss below) and the internal differential quantum efficiency $\eta_{\text {int }}$, an important parameter describing the efficiency of stimulated recombination. The standard procedure ${ }^{2}$ of determining $\alpha_{\text {int }}$ is used to extract $\eta_{\text {int }}$ as well. I discuss here the practicality of this procedure for quantum dot (QD) lasers. Self-organized QDs have attracted considerable recent attention as a new class of materials for a wide spectrum of applications. ${ }^{3}$

\section{ANALYSIS AND DISCUSSION}

The output optical power of a diode laser is $P$ $=(\hbar \omega / e)\left(I-I_{\mathrm{th}}\right) \eta_{\mathrm{int}} \eta_{\mathrm{opt}}$, where $\hbar \omega$ is the photon energy, $e$ is the electron charge, $I$ is the injection (pump) current, $I_{\text {th }}$ is the threshold current, and $\eta_{\text {opt }}$ is the optical efficiency of the laser cavity. For light emitted from both edges of a FabryPerot cavity, $\eta_{\mathrm{opt}}=\beta /\left(\beta+\alpha_{\mathrm{int}}\right)$, where $\beta=(1 / L) \ln (1 / R)$ is the mirror loss, $L$ is cavity length, and $R$ is the facet reflectivity.

The slope (or external differential quantum) efficiency is

$$
\eta_{\mathrm{ext}}=\frac{1}{(\hbar \omega / e)} \frac{\partial P}{\partial I}=\left[1+\left(I-I_{\mathrm{th}}\right) \frac{\partial \ln \left(\eta_{\mathrm{int}} \eta_{\mathrm{opt}}\right)}{\partial I}\right] \eta_{\mathrm{int}} \eta_{\mathrm{opt}} \text {. }
$$

Both $\eta_{\text {int }}$ and $\eta_{\text {opt }}$ decrease with the pump current. The decrease of $\eta_{\text {int }}$ arises from the following (see Ref. 4): (i) noninstantaneous carrier capture into the quantum-confined active region, and (ii) superlinear (in the carrier density) recombination rate outside the active region. Because of (i), the carrier density outside the active region rises with injection current $I$, even above threshold, and because of (ii), the

\footnotetext{
${ }^{a)}$ Electronic mail: asryan@mse.vt.edu. URL: http://www.mse.vt.edu/people/ faculty/asryan.html
}

useful fraction of current (that ends up as output light) decreases. The decrease of $\eta_{\text {opt }}$ arises from the increase of $\alpha_{\text {int }}{ }^{5}$ caused by the carrier density rise with $I$.

Hence the product of $\eta_{\text {int }}$ and $\eta_{\text {opt }}$ does not present $\eta_{\text {ext }}$ for $I>I_{\text {th }}$. Only at the lasing threshold $\left(I=I_{\text {th }}\right), \quad \eta_{\text {ext,th }}$ $=\eta_{\text {int,th }} \eta_{\mathrm{opt} \text {,th }}$, and the reciprocal of $\eta_{\mathrm{ext} \text {,th }}$ is

$$
\frac{1}{\eta_{\mathrm{ext}, \mathrm{th}}}=\frac{1}{\eta_{\mathrm{int}, \mathrm{th}}}\left(1+\frac{\alpha_{\mathrm{int}, \mathrm{th}}}{\ln \frac{1}{R}} L\right),
$$

where the subscript "th" denotes the quantities at the lasing threshold.

The standard method of determining $\alpha_{\text {int,th }}$ and $\eta_{\text {int,th }}$ is based on an often implicit assumption that they do not change with $L$. Provided such is the case, a measured plot of $1 / \eta_{\text {ext,th }}$ vs $L$ is an inclined straight line [see Eq. (2)]. The intersection of this line with the $y$ axis gives $1 / \eta_{\text {int,th }}$, and the incline yields $\alpha_{\text {int,th }}$ (since $R$ is known).

The assumption of constant $\alpha_{\text {int,th }}$ and $\eta_{\text {int,th }}$, which are independent of $L$, is, however, not justified in the general case. This has been discussed previously in the context of multiple-quantum-well (QW) lasers (see, for instance, Ref. 6). Here, I discuss the standard cavity-length method in QD lasers.

In Ref. 7, a strong (critical) dependence of $\eta_{\text {int }}$ on $L$ was described in semiconductor lasers with a quantum-confined active region $-\eta_{\text {int }}$ drops to zero as $L$ reduces and approaches the shortest cavity length $L^{\mathrm{min}}$, at which the lasing is attainable. For $L$ well above $L^{\text {min }}, \eta_{\text {int }}$ increases and saturates at unity; hence the assumption on constant $\eta_{\text {int,th }}$ can only be justified in long-cavity devices. The $L$-dependence of $\eta_{\text {int }}$ in itself places limitations on the standard procedure in short-cavity devices.

In this paper, I quantitatively analyze the limitations placed on the standard method by the $L$-dependence of $\alpha_{\text {int,th }}$ in QD lasers. Correspondingly, a plot of the reciprocal optical efficiency at the lasing threshold, $1 / \eta_{\text {opt,th }}$, versus $L$ will be analyzed.

In general, $\alpha_{\text {int }}$ increases with the carrier density. ${ }^{5}$ At the lasing threshold, 


$$
\alpha_{\text {int,th }}=\alpha_{0}+\sigma_{\text {int }} n_{\text {th }},
$$

where $\alpha_{0}$ is a constant, $\sigma_{\text {int }}$ is an effective cross section for the absorption loss processes, ${ }^{5}$ and $n_{\text {th }}$ is the carrier density (outside or inside the active region-see below).

The $L$-dependence of $\alpha_{\text {int,th }}$ comes from such a dependence of $n_{\mathrm{th}}$. Indeed, the lasing threshold condition reads as

$$
g\left(n_{\mathrm{th}}\right)=\frac{1}{L} \ln \frac{1}{R}+\alpha_{0}+\sigma_{\mathrm{int}} n_{\mathrm{th}},
$$

where $g$ is the peak value of the modal gain spectrum, which depends on $n_{\text {th }}$.

The functional dependence of $n_{\text {th }}$ on $L$ is determined by the type of active region, i.e., by the functional dependence of $g$ on $n_{\text {th }}$, and by the dominant mechanism of internal loss. The focus of this paper is on a QD laser. For reference, a QW laser will also be considered.

Consider first a QD laser with the carrier-densitydependent internal loss outside its active region. Such loss [e.g., free-carrier or intervalence-band absorption in the optical confinement layer (OCL) or in the wetting layer] is typical in a QD laser. At the same time, the internal loss in the active region, i.e., in QDs themselves, is low. ${ }^{8}$ Assuming equilibrium distribution of carriers in QDs and in the OCL [relatively high temperatures $T>T_{g}$, where $T_{g}$ is given by Eq. (26) in Ref. 8] and using the corresponding equation for the gain [Eq. (6) in Ref. 9], Eq. (4) becomes

$$
g_{\mathrm{QD}} \frac{n_{\mathrm{th}}-n_{1}}{n_{\mathrm{th}}+n_{1}}=\frac{1}{L} \ln \frac{1}{R}+\alpha_{0}+\sigma_{\mathrm{int}} n_{\mathrm{th}},
$$

where $g_{\mathrm{QD}}^{\max }$ is the maximum (saturation) value of the modal gain [given by Eq. (20) in Ref. 9], $n_{1}=N_{c}^{3 \mathrm{D}} \exp \left(-E_{n} / T\right)$, $N_{c}^{3 \mathrm{D}}=2\left(m_{c}^{\mathrm{OCL}} T / 2 \pi \hbar^{2}\right)^{3 / 2}$ is the three-dimensional (3D) effective density of states in the OCL, the temperature $T$ is measured in units of energy, and $E_{n}$ is the carrier excitation energy from a QD. The quantity $n_{1}$ characterizes the intensity of the thermal escape (leakage) of carriers from a QD. Here, $n_{\text {th }}$ is a $3 \mathrm{D}$ carrier density in the OCL measured in $\mathrm{cm}^{-3}$, and $\sigma_{\text {int }}$ is measured in $\mathrm{cm}^{2}$, so that $\sigma_{\text {int }} n_{\text {th }}$ is measured in $\mathrm{cm}^{-1}$.

Consider now a QW laser with the carrier-densitydependent internal loss in its active region. Such loss (e.g., intervalence-band absorption) is typical in QWs. ${ }^{10}$ In fact, the carrier-density-dependent absorption within QWs can be the dominant mechanism for internal optical loss in $1.55 \mu \mathrm{m}$ InGaAsP QW lasers. ${ }^{6}$ Using the model for the gain, ${ }^{11,12}$ which neglects the intraband relaxation broadening, Eq. (4) becomes

$$
\begin{aligned}
g_{\mathrm{QW}}^{\max } & {\left[1-\exp \left(-\frac{n_{\mathrm{th}}}{N_{c}^{2 \mathrm{D}}}\right)-\exp \left(-\frac{m_{c}}{m_{v}} \frac{n_{\mathrm{th}}}{N_{c}^{2 \mathrm{D}}}\right)\right] } \\
= & \frac{1}{L} \ln \frac{1}{R}+\alpha_{0}+\sigma_{\mathrm{int}} n_{\mathrm{th}},
\end{aligned}
$$

where $g_{\mathrm{QW}}^{\max }$ is the maximum (saturation) value of the modal gain [see Refs. 11 and 12, and Eq. (9) in Ref. 13], $N_{c}^{2 \mathrm{D}}$ $=m_{c} T /\left(\pi \hbar^{2}\right)$ is the $2 \mathrm{D}$ effective density of states for the conduction band in a $\mathrm{QW}$, and $m_{c}$ and $m_{v}$ are the electron and hole effective masses in a QW. Here, $n_{\text {th }}$ is a $2 \mathrm{D}$ carrier
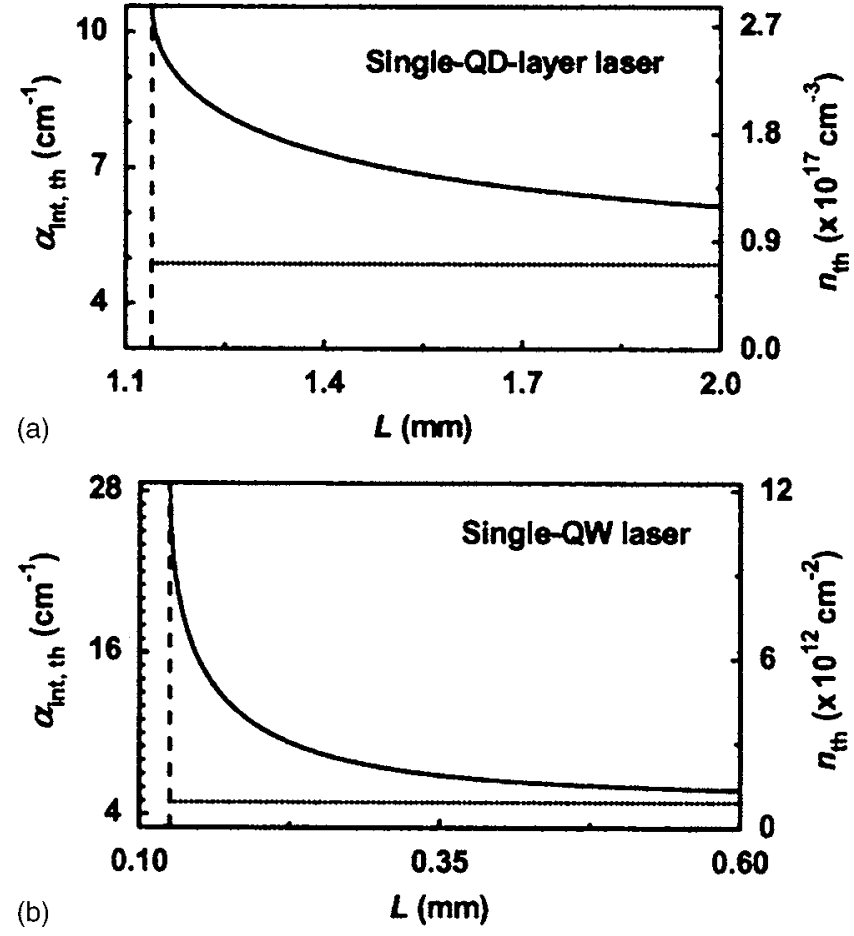

FIG. 1. Carrier density (right axis) and internal loss (left axis) at the lasing threshold vs the cavity length in a single-QD-layer laser (a) and single-QW laser (b). Horizontal dotted lines show $n_{\text {th }}$ and $\alpha_{\text {int,th }}$ in an infinitely long cavity. Vertical dashed lines mark the shortest cavity length $L^{\mathrm{min}}$ at which the lasing is attainable. Throughout the paper, GaInAsP/InP-based QW (Ref. 13) and QD (Ref. 8) lasers are considered for illustration. As-cleaved facet reflectivity at both ends $(R=0.32)$ and room-temperature operation are assumed. Both in QW and QD lasers, $\alpha_{0}=3 \mathrm{~cm}^{-1}$ and $\alpha_{\text {asympt }}=4.88 \mathrm{~cm}^{-1}$. The QW laser parameters in Eq. (6) are as follows: $g_{\mathrm{OW}}^{\max }=130.17 \mathrm{~cm}^{-1}, N_{c}^{2 \mathrm{D}}$ $=4.45 \times 10^{11} \mathrm{~cm}^{-2}, m_{v} / m_{c}=10.98$, and $\sigma_{\text {int }}=2.09 \times 10^{-12} \mathrm{~cm}$. The QD laser parameters in Eq. (5) are as follows: $g_{\mathrm{QD}}^{\max }=29.52 \mathrm{~cm}^{-1}, \quad n_{1}=5.07$ $\times 10^{16} \mathrm{~cm}^{-3}$, and $\sigma_{\text {int }}=2.65 \times 10^{-17} \mathrm{~cm}^{2}$.

density measured in $\mathrm{cm}^{-2}$, and $\sigma_{\text {int }}$ is measured in $\mathrm{cm}$, so that $\sigma_{\text {int }} n_{\text {th }}$ is measured in $\mathrm{cm}^{-1}$.

Hence finding $n_{\mathrm{th}}(L)$ requires the solution of the algebraic Eq. (5) or (6). Equation (5) is actually a quadratic equation. 5

Figure 1 shows $n_{\text {th }}(L)$ in single-QD-layer (a) and single-QW (b) lasers. Both in QD and QW lasers, $n_{\text {th }}$ increases steeply with reducing $L$ and approaching the shortest cavity length $L^{\mathrm{min}}$, at which the threshold condition [Eq. (6) or (5)] is satisfied. $L^{\mathrm{min}}$ in a QW laser $(125 \mu \mathrm{m})$ is considerably shorter than that in a QD laser $(1.139 \mathrm{~mm})$. This is because QDs cover only a small surface fraction of the active layer and hence the maximum modal gain of a single-QDlayer laser is smaller $\left(g_{\mathrm{QD}}^{\max }=29.5 \mathrm{~cm}^{-1}\right)$ than that of a single-QW laser $\left(g_{\mathrm{QW}}^{\max }=130 \mathrm{~cm}^{-1}\right)$.

In long cavities, $n_{\text {th }}$ reduces and asymptotically approaches a constant value corresponding to the "no mirror loss" $(L=\infty)$ case (horizontal dotted lines in Fig. 1). In the absence of internal loss, this value of $n_{\text {th }}$ would go into the carrier density at the transparency threshold.

The dependence of $\alpha_{\text {int,th }}$ on $L$ is obtained from $n_{\text {th }}(L)$ by a simple rescaling [see Eq. (3)] and is also shown in Fig. 1. Horizontal dotted lines show the internal loss in an infinitely long cavity, $\alpha_{\text {asympt }}$. The internal loss is significantly elevated in short cavities. 

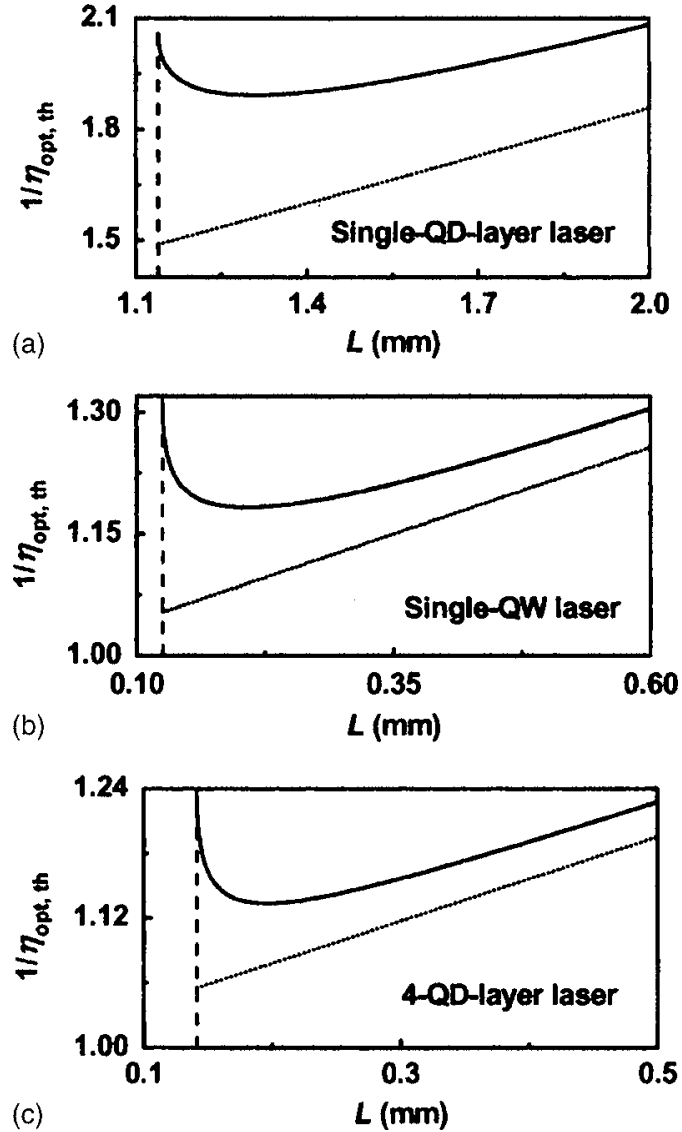

FIG. 2. Reciprocal optical efficiency at the lasing threshold vs the cavity length in a single-QD-layer laser (a), single-QW laser (b), and four-QDlayer laser (c). Inclined straight dotted lines show $1 / \eta_{\text {opt,th }}$ assuming $\alpha_{\text {int, th }}$ $=$ const $(L)=\alpha_{\text {asympt }}$. Vertical dashed lines mark the shortest cavity length $L^{\text {min }}$ at which the lasing is attainable. Parameters of single-QW and single-QDlayer lasers are the same as in Fig. 1. For a four-QD-layer laser, $g_{\mathrm{QD}}^{\max }$ $=118.08 \mathrm{~cm}^{-1}$.

Figure 2 shows $1 / \eta_{\text {opt,th }}$ vs $L$ in single-QD-layer (a), single-QW (b), and four-QD-layer (c) lasers. The inclined straight dotted lines show $1 / \eta_{\text {opt,th }}$ assuming $\alpha_{\text {int,th }}$ $=\operatorname{const}(L)=\alpha_{\text {asympt }}$. Throughout the entire range of $L$ shown, the actual $1 / \eta_{\mathrm{opt}, \text { th }}$ is higher than that assuming $\alpha_{\mathrm{int}, \text { th }}$ $=\operatorname{const}(L)$. Of special note is the nonmonotonic dependence of $1 / \eta_{\mathrm{opt}, \text { th }}$ on $L$. With reducing $L$, the curve for $1 / \eta_{\mathrm{opt}, \text { th }}$ never intersects the $y$ axis; instead, for $L$ below a certain value, the increase in $\alpha_{\text {int,th }}$ is steeper than the decrease in $L$ so that the product of $\alpha_{\text {int,th }}$ and $L$ [see Eq. (2)] increases and

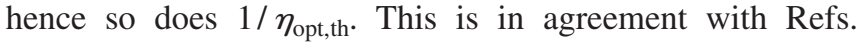
14-16, where such nonmonotonic dependence on $L$ was observed experimentally for QD lasers (cf. Fig. 7 in Ref. 14, Fig. 1 in Ref. 15, and Fig. 2 in Ref. 16). At the shortest cavity length at which the lasing is attainable in the structure, the difference between the actual $1 / \eta_{\mathrm{opt} t \text { th }}$ and that assuming $\alpha_{\text {int,th }}=\operatorname{const}(L)$ is at its maximum.

As $\alpha_{\text {int,th }}$ asymptotically approaches $\alpha_{\text {asympt }}$ with increasing $L$ (dotted line, Fig. 1), $1 / \eta_{\text {opt,th }}$ asymptotically approaches its value assuming $\alpha_{\text {int,th }}=\alpha_{\text {asympt }}$ (dotted line, Fig. 2).

The above character of the plot of $1 / \eta_{\text {opt,th }}$ (and hence of $\left.1 / \eta_{\text {ext,th }}\right)$ vs $L$ should be inherent in any diode laser with the carrier-density-dependent $\alpha_{\text {int }}$. However, as seen from Fig. 2,

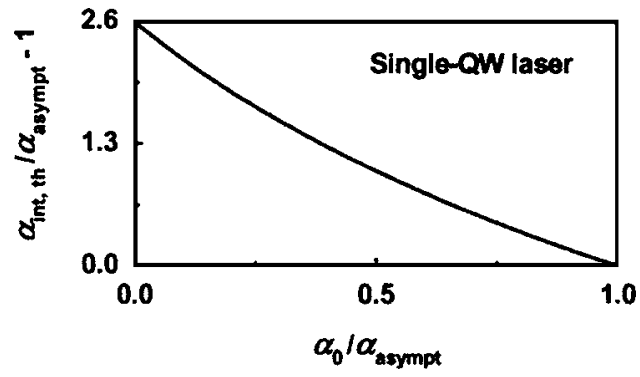

FIG. 3. Relative difference between the internal losses in $250 \mu \mathrm{m}$ and infinitely long cavity single-QW lasers, $\left(\alpha_{\text {int,th }}-\alpha_{\text {asympt }}\right) / \alpha_{\text {asympt }}$, vs the constant component of the internal loss $\alpha_{0}$ normalized to $\alpha_{\text {asympt }} \cdot \alpha_{\text {asympt }}=4.88 \mathrm{~cm}^{-1}$.

the nonmonotonic change in $1 / \eta_{\text {opt,th }}$ occurs at relatively short $L$; accordingly, the limitations on the standard method are strong at such $L$. The higher $g^{\max }$ and/or the lower $\alpha_{0}$ and $\sigma_{\text {int }}$, the shorter can be the cavity in which the lasing is attainable [see (6) and (5)]. Hence, increasing $g^{\max }$ shifts the nonmonotonic portion of $1 / \eta_{\text {opt,th }}$ toward lower $L$. This is demonstrated in Fig. 2. In a single-QD-layer laser (a), $g_{\mathrm{QD}}^{\max }$ $=29.5 \mathrm{~cm}^{-1}$, and the shortest cavity length is above $1 \mathrm{~mm}$ $\left(L_{\mathrm{QD}}^{\mathrm{min}}=1.139 \mathrm{~mm}\right)$. Hence the limitations are strong over the entire range of $L$. In a single-QW laser (b), $g_{\mathrm{QW}}^{\max }=130 \mathrm{~cm}^{-1}$ and $L_{\mathrm{QW}}^{\min }=125 \mu \mathrm{m}$. In a four-QD-layer laser (c), $g_{\mathrm{QD}}^{\max }$ $=118 \mathrm{~cm}^{-1}$ is close to that in a single-QW laser, and hence $L_{\mathrm{QD}}^{\min }=141 \mu \mathrm{m}$ is close to that in a single-QW laser; correspondingly, the plots for $1 / \eta_{\text {opt,th }}$ are similar in these cases. In a multiple-QW laser, $L^{\mathrm{min}}$ will be below $100 \mu \mathrm{m}$, which is to say the limitations on the standard procedure can apply only at such short $L$.

Another factor, strongly affecting $1 / \eta_{\mathrm{opt}, \text { th }}$, is how $\alpha_{\text {int,th }}$ is distributed over its constant and carrier-density-dependent components [the first and the second terms on the right-hand side of (3), respectively]. This is demonstrated in Fig. 3 for a single-QW laser, where the relative difference between the actual $\alpha_{\text {int,th }}$ at a given cavity length $(L=250 \mu \mathrm{m})$ and that in an infinitely long cavity, $\left(\alpha_{\text {int,th }}-\alpha_{\text {asympt }}\right) / \alpha_{\text {asympt }}$, is shown versus the constant component $\alpha_{0}$ normalized to $\alpha_{\text {asympt }}$. The difference is large at $\alpha_{0}=0$. Naturally, the difference vanishes if the internal loss is comprised of only a constant component. Hence in those structures, in which $\alpha_{\text {int,th }}$ is predominantly constant (independent of the carrier density), e.g., it is caused by scattering at rough interfaces, the conventional procedure applies also to short cavities.

\section{CONCLUSIONS}

In conclusion, the curve for $1 / \eta_{\text {ext,th }}$ vs $L$ in a QD laser can qualitatively deviate from an inclined straight line assumed in the conventional method for determining $\eta_{\text {int,th }}$ and $\alpha_{\text {int,th }}$; particularly, $1 / \eta_{\text {ext,th }}$ increases with reducing $L$ in short cavities. Only "no mirror loss" $(L=\infty)$ values of $\alpha_{\text {int,th }}$ and $\eta_{\text {int,th }}$ can be extracted from the measurement of $1 / \eta_{\text {ext,th }}$ vs $L$; these values will be provided by the inclined straight line presenting the asymptote for the actual curve for $1 / \eta_{\text {ext,th }}(L)$ at $L \rightarrow \infty$.

For $L$ longer than several hundred $\mu \mathrm{m}$, the limitations on 
the standard procedure are strong in a single-QD-layer laser, moderate in single-QW and multiple-QD-layer lasers, and slight in a multiple-QW laser.

While only the effect of internal loss has been included in the model for the calculations, it has been discussed in the paper that the $L$-dependence of the internal quantum efficiency affects $1 / \eta_{\text {ext,th likewise, thus making even stronger }}$ the deviation of the plot of $1 / \eta_{\text {ext,th }}$ vs $L$ from a straight line. The same is true of the other effects not considered here. Thus, for instance, the carrier density, and hence the internal loss, will be increased due to the heating effect.

\section{ACKNOWLEDGMENT}

This work was supported by the U.S. Army Research Office under Grant No. W911-NF-05-1-0308.

${ }^{1}$ Quantum Well Lasers, edited by P. S. Zory, Jr. (Academic, Boston, 1993), p. 504.

${ }^{2}$ L. A. Coldren and S. W. Corzine, "Diode Lasers and Photonic Integrated Circuits (Wiley, New York, 1995), p. 594.

${ }^{3}$ D. Bimberg, J. Phys. D 38, 2055 (2005).
${ }^{4}$ L. V. Asryan, S. Luryi, and R. A. Suris, Appl. Phys. Lett. 81, 2154 (2002). ${ }^{5}$ L. V. Asryan and S. Luryi, Appl. Phys. Lett. 83, 5368 (2003).

${ }^{6}$ J. Piprek, P. Abraham, and J. E. Bowers, IEEE J. Sel. Top. Quantum Electron. 5, 643 (1999).

${ }^{7}$ L. V. Asryan, S. Luryi, and R. A. Suris, IEEE J. Quantum Electron. 39, 404 (2003)

${ }^{8}$ L. V. Asryan and R. A. Suris, Semicond. Sci. Technol. 11, 554 (1996).

${ }^{9}$ L. V. Asryan and S. Luryi, IEEE J. Quantum Electron. 40, 833 (2004).

${ }^{10}$ M. Asada, A. Kameyama, and Y. Suematsu, IEEE J. Quantum Electron. 20, 745 (1984).

${ }^{11}$ K. J. Vahala and C. E. Zah, Appl. Phys. Lett. 52, 1945 (1988).

${ }^{12} \mathrm{~S}$. L. Chuang, Physics of Optoelectronic Devices (Wiley, New York, 1995), p. 717.

${ }^{13}$ L. V. Asryan, N. A. Gun'ko, A. S. Polkovnikov, G. G. Zegrya, R. A. Suris, P.-K. Lau, and T. Makino, Semicond. Sci. Technol. 15, 1131 (2000).

${ }^{14}$ M. V. Maximov, L. V. Asryan, Yu. M. Shernyakov, A. F. Tsatsul'nikov, I. N. Kaiander, V. V. Nikolaev, A. R. Kovsh, S. S. Mikhrin, V. M. Ustinov, A. E. Zhukov, Zh. I. Alferov, N. N. Ledentsov, and D. Bimberg, IEEE J. Quantum Electron. 37, 676 (2001).

${ }^{15}$ A. E. Zhukov, A. R. Kovsh, V. M. Ustinov, and Zh. I. Alferov, Laser Phys. 13, 319 (2003).

${ }^{16}$ A. R. Kovsh, N. A. Maleev, A. E. Zhukov, S. S. Mikhrin, A. P. Vasil'ev, E. A. Semenova, Yu. M. Shernyakov, M. V. Maximov, D. A. Livshits, V. M. Ustinov, N. N. Ledentsov, D. Bimberg, and Zh. I. Alferov, J. Cryst. Growth 251, 729 (2003). 